AFCRC-TN-55-795

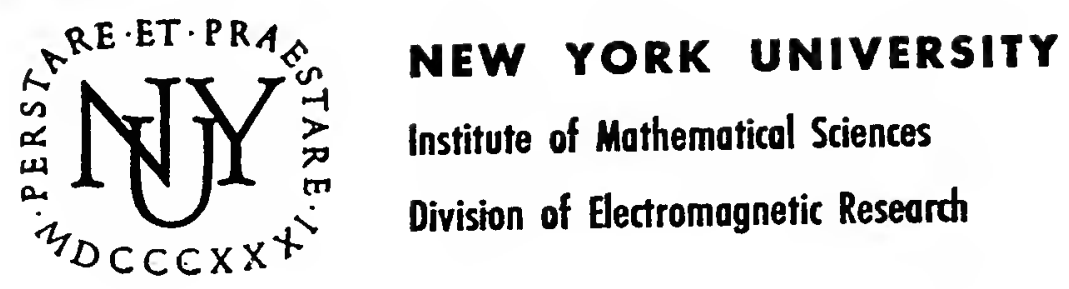

RESEARCH REPORT No. EM-82

\title{
Calculation of Charge Density Distribution of Multilayers from Transit Time Data
}

SAMUEL N. KARP and JERRY SHMOYS

CONTRACT NO. AF $19(122)-42$

JULY 1955 

NEW YORK UNIVERSITY

Institute of Mathematical Sciences

Division of Electromagnetic Research

Research Report No. EM-82

\section{CALCULATION OF CHARGE DENSITY DISTRIBUTION \\ OF MULTILAYERS FROM TRANSIT TIME DATA}

Samuel N. Karp and Jerry Shmoys

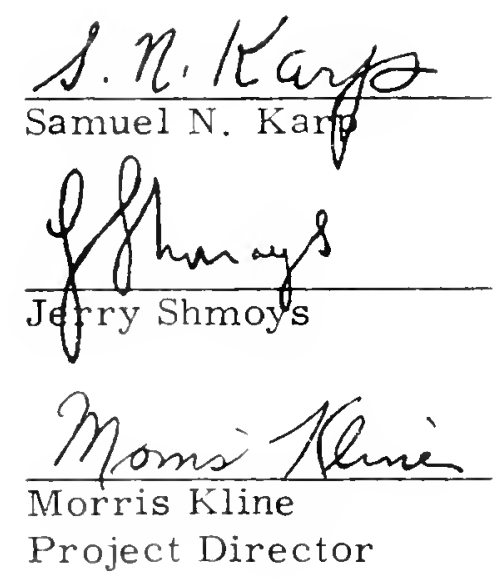

The research reported in this document has been made possible through support and sponsorship extended by the Air Force Cambridge Research Center, under Contract Nc. AF-19(122)-42. It is published for technical information only and does not necessarily represent recommendations or conclusions of the sponsoring agency. 

An integral relation is derived between the electron density distribution of a multilayer and the frequency dependence of the time in which a radio pulse traverses the layer. This integral equation is then sclined for the electron distribution by means of the Mellin transform, given the transit time function. The lack of uniqueness of this solution is discussed. Certain characteristics of the multilayer, $e . g$. the minimum electron density, are given in terms of the Mellin transform of the transit time.

The same procedure is applied to the problem of calculating the potentfal of a potential well from the transit time of a particle as a function of energy.

\section{Table of Contents}

Page

1. Introduction

1

2. The integral equation

3. Solution of the integral equation

4. Special values

5. Conciusion

Appendix: Motion of a particle in a potential well 
1. Introduction

Almost as soon as the existence of the ionosphere was accepted as a fact, there arose the problem of determining the ionization density as a function of height. The method of sounding the ionosphere by short pulses of radio waves was developed. From the time of travel of such a pulse as a function of frequency, the electron density as a function of height can be inferred. One way to do this is to assume a type of density distribution (such as a parabolic layer), and then

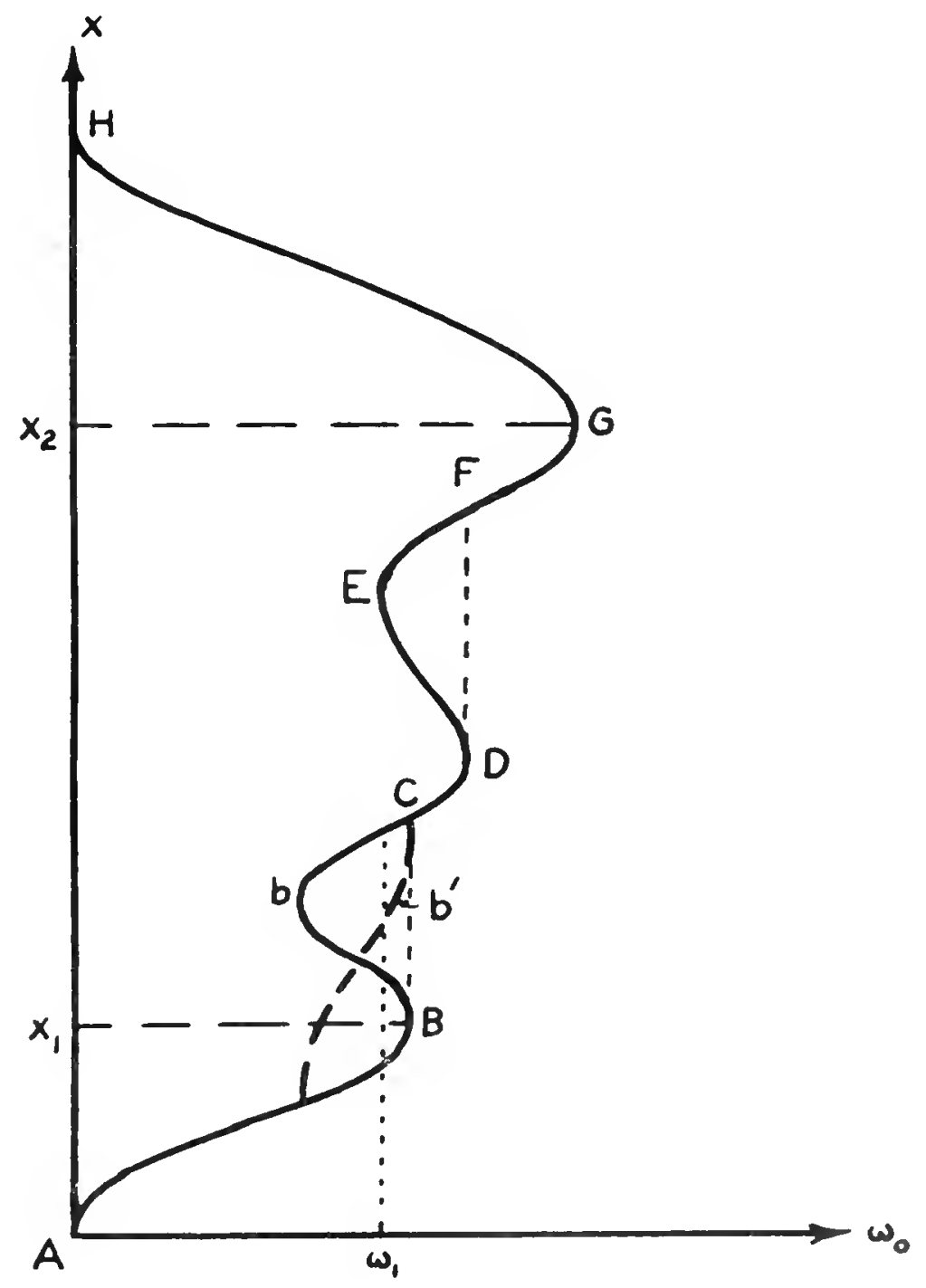


determine the parameters characterizing such a distribution by requiring the theoretical curve for such a distribution to agree with the experimental one at a number of points. Another way, more important for the purposes of this report, is to treat the integral relation between the travel time function and the electron density function as an integral equation. If we neglect the effect of the magnetic field, then this integral equation can be solved [l] provided the electron density is a monotonically increasing function of height.

The actual electron density distribution of the lonosnhere is not monotonic: It contains several peaks and valleys (see Fig. 1). Manning made an estimate of the error introduced in the calculated distribution by assuming constant density in the valleys. In this way one can calculate approximately (but with known error) the portions $A B, C D$, and $F G$ of the curve from the time of travel of the pulse from the bottom of the layer, $A$, to the point of reflection and back to $A$. In such a calculation one makes use of the travel time as a function of frequency from zero frequency to the maximum critical frequency of the ionosphere. Above this frequency the pulse is no longer reflected by the ionosphere.

In this paper we shall assume that the portions $A B$ and $G H$ of $F i g \cdot I$ are known from soundings from below and above, and we shall concern ourselves with the calculation of the distribution $B G$ from the time of travel from $x_{1}$ to $x_{2}$ at frequencies higher than the critical frequency at $x_{2}$. We shall utilize information about the transit time of a radio pulse through the ionosphere, from $A$ to $\mathrm{H}$, at frequencies above the maximum critical frequency of the ionosphere. In order to obtain such information we would, of course, have to have a variable frequency transmitter above the ionosphere, say in a rocket. Alternatively, referring to the type of distribution shown on Fig. 2, if the density in the region CD above $x_{2}$ is assumed known, our method could be applied. In this case, using sounding information we can obtain the time of travel from $x_{1}$ to $x_{2}$, from which we shall obtain information about the distribution $\mathrm{BC}$. 


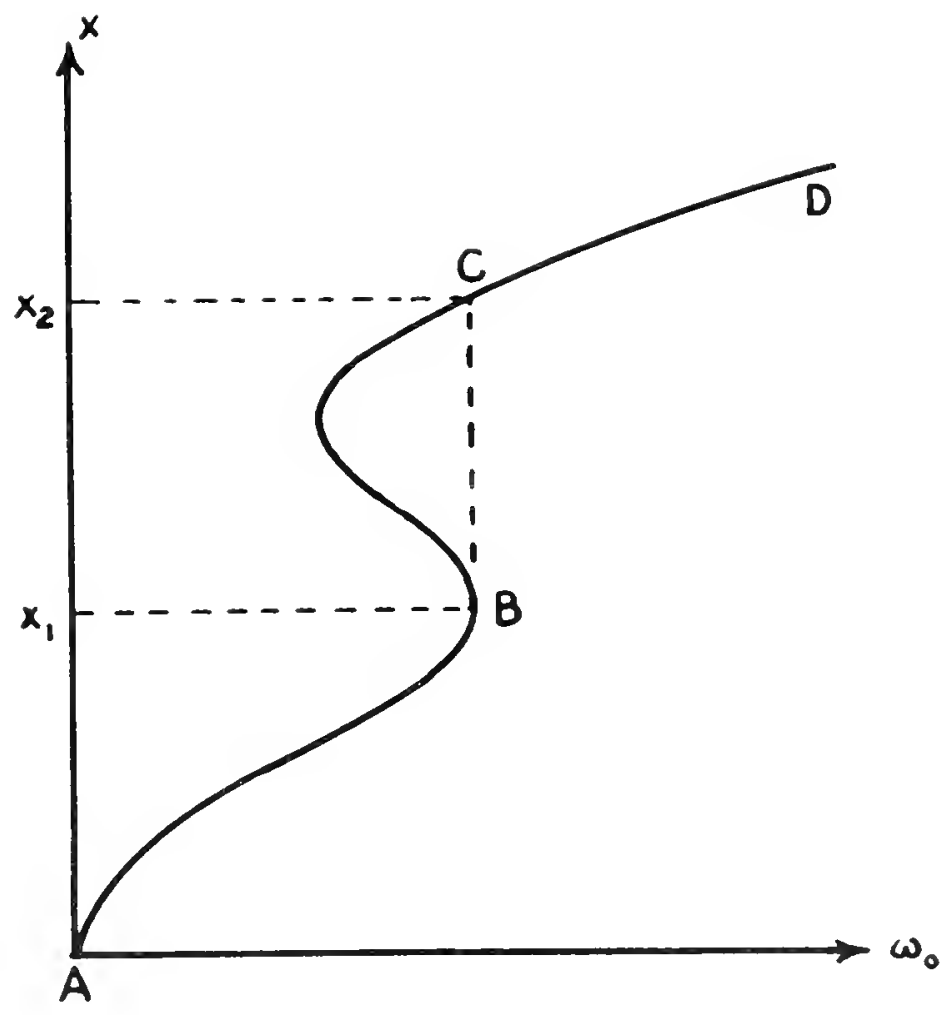

\section{Fisure 2}

2. The integral equation

A radlo pulse of carrier frequency $w$ travels in the lonosphere with velocity

(1)

$$
\nabla=c\left(1-\omega_{0}^{2} / \omega^{2}\right)^{1 / 2} \text {, }
$$

where $c$ is the velocity of light and $\omega_{0}$ is the plasma frequency or critical frequency of the ionized medium. The latter is related to the electron density $\mathrm{N}$, and to the charge and mass of the electron, $e$ and $m$, as follows:

$$
\omega_{0}^{2}=4 \pi e^{2} m^{-1} c^{2} N
$$

In the case of ionospheric sounding the pulse is reflected at the height ${ }^{x}$, at which its velocity goes to zero, so that
(3)
$\omega_{0}\left(x_{r}\right)=\omega$. 
The time of travel of the pulse to the reflection point and back is

$$
\tau(\omega)=2 \int_{x_{0}}^{x_{r}(\omega)} \frac{d x}{v(x, \omega)}=\frac{2}{c} \int_{x_{0}}^{x_{r}(\omega)}\left[1-\omega_{0}^{2}(x) / \omega^{2}\right]^{-1 / 2} d x,
$$

where $x_{0}$ is the height of the transmitter. If in the above equation, we are given $\tau(\omega)$ and we are told that $\omega_{0}(x)$ is monotonically increasing with $x$, then we can solve this Abel type integral equation. The function we obtain in such a solution is not $\omega_{0}(x)$ but its inverse, $x\left(\omega_{0}\right)$ :

$$
x\left(\omega_{0}\right)=x_{0}+(c / \pi) \int_{0}^{\pi / 2} \tau\left(\omega_{0} \sin \xi\right) d \xi .
$$

In our case we are given the time of travel between two fixed points, rather than between a fixed point and a variable one $\left(x_{r}\right)$; thus we have

$$
\tau_{12}(\omega)=\int_{x_{1}}^{x_{2}} \frac{d x}{v(x, \omega)}=\frac{1}{c} \int_{x_{1}}^{x_{2}}\left[1-\frac{\omega_{0}^{2}(x)}{\omega^{2}}\right]^{-i / 2} d x .
$$

If we examine this expression, we find that more than one $\omega_{0}(x)$ will yield the same $\tau_{12}(\omega)$. For example, if same $\omega_{0}(x)$ yields a given $\tau(\omega)$, then $\omega_{0}\left(x_{2}+x_{1}-x\right)$ will also yield the same $\tau(\omega)$ [unless $\omega_{0}(x)$ is symmetric about $\frac{1}{2}\left(x_{1}+x_{2}\right)$ it is not identical to $\left.\omega_{0}\left(x_{2}+x_{1}-x\right)\right]$. There is actually an infinite number of functions $\omega_{0}(x)$ which yield the same transit time. In order to show this, let us consider first the relatively simple case of a double layer, $1 . e .$, the case in which $\omega_{0}(x)$ has maxima at $x_{1}$ and $x_{2}$ only and a single minimum at $x_{3}$, at which $1 t$ has the value $\omega_{3}$ (see Fig. 3). Further, let us denote by $x_{4}$ the point at which the critical frequency is equal to $\omega_{1}$ (or $\omega_{2}$, if $\left.\omega_{2}<\omega_{1}\right)$. The function $x\left(\omega_{0}\right)$ is then doublevalued in the range $\omega_{3}<\omega \leq \omega_{1}$. Let us denote the upper branch of this function by $x_{u}(\omega)$, and the lower one by $x_{l}(\omega)$. For convenience, let us define these 
$-5-$

functions for higher values of $\omega:$

(7)

$$
\omega \geq \omega_{1}, \quad x_{\ell}(\omega)=x_{1}
$$

$$
\omega \geq \omega_{2}, \quad x_{u}(\omega) \equiv x_{2}
$$

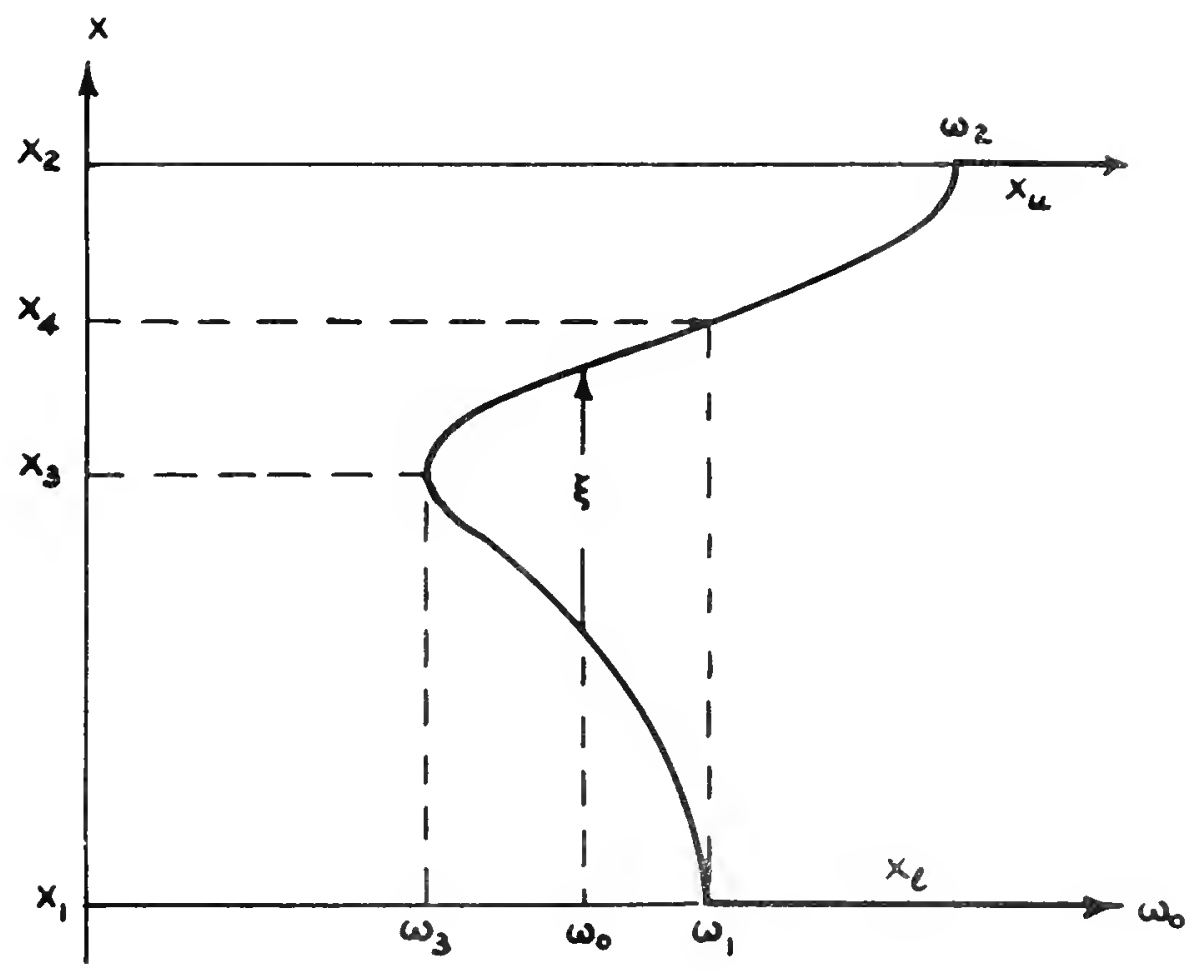

Figure 3

We can split the integral for transit time into two parts, as follows:

(8) $\tau_{12}(\omega)=\tau_{13}+\tau_{32}=\frac{1}{c} \int_{x_{1}}^{x_{3}}\left[1-\left(\omega_{0} / \omega\right)^{2}\right]^{-1 / 2} d x+\frac{1}{c} \int_{x_{3}}^{x_{2}}\left[1-\left(\omega_{0} / \omega\right)^{2}\right]^{-1 / 2} d x$.

In each of these we can introduce $y=\omega_{0}^{2}$ as the variable of integration, so that 
$-6-$

$$
d x=(d x / d y) d y=\left(d x / d \omega_{0}^{2}\right) d y
$$

and

$$
\begin{aligned}
& \tau_{12}(\omega)=\frac{1}{c} \int_{\omega_{1}}^{\omega_{3}}\left[1-(y / \omega)^{2}\right]^{-1 / 2} \frac{d x^{(y)}}{d y} d y+\frac{1}{c} \int_{\omega_{3}}^{\omega_{2}}\left[1-(y / \omega)^{2}\right]^{-1 / 2 \frac{d x}{u}(y)} \frac{d y}{d y} \\
&=\frac{1}{c} \int_{\omega_{3}}^{\infty}\left[1-(y / \omega)^{2}\right]^{-1 / 2}\left[\frac{d x}{d y}-\frac{d x}{d y}\right] d y \\
&=\frac{1}{c} \int_{\omega_{3}}^{\infty}\left[1-(y / \omega)^{2}\right]^{-1 / 2} \frac{d \xi}{d y} d y, \\
& \xi=x_{u}-x_{l} .
\end{aligned}
$$

(9)

In the above manipulation we have assumed that $x\left(\omega_{0}\right)$ is sufficiently well behaved. We see that the transit time is the same for ali valleys whose width $\xi$ is the same function of $\omega_{0}$. In a more complicated ionospheric structure, consisting of more than two layers, $\xi$ is the sum of the widths of all the valleys (see Fig. 4). If

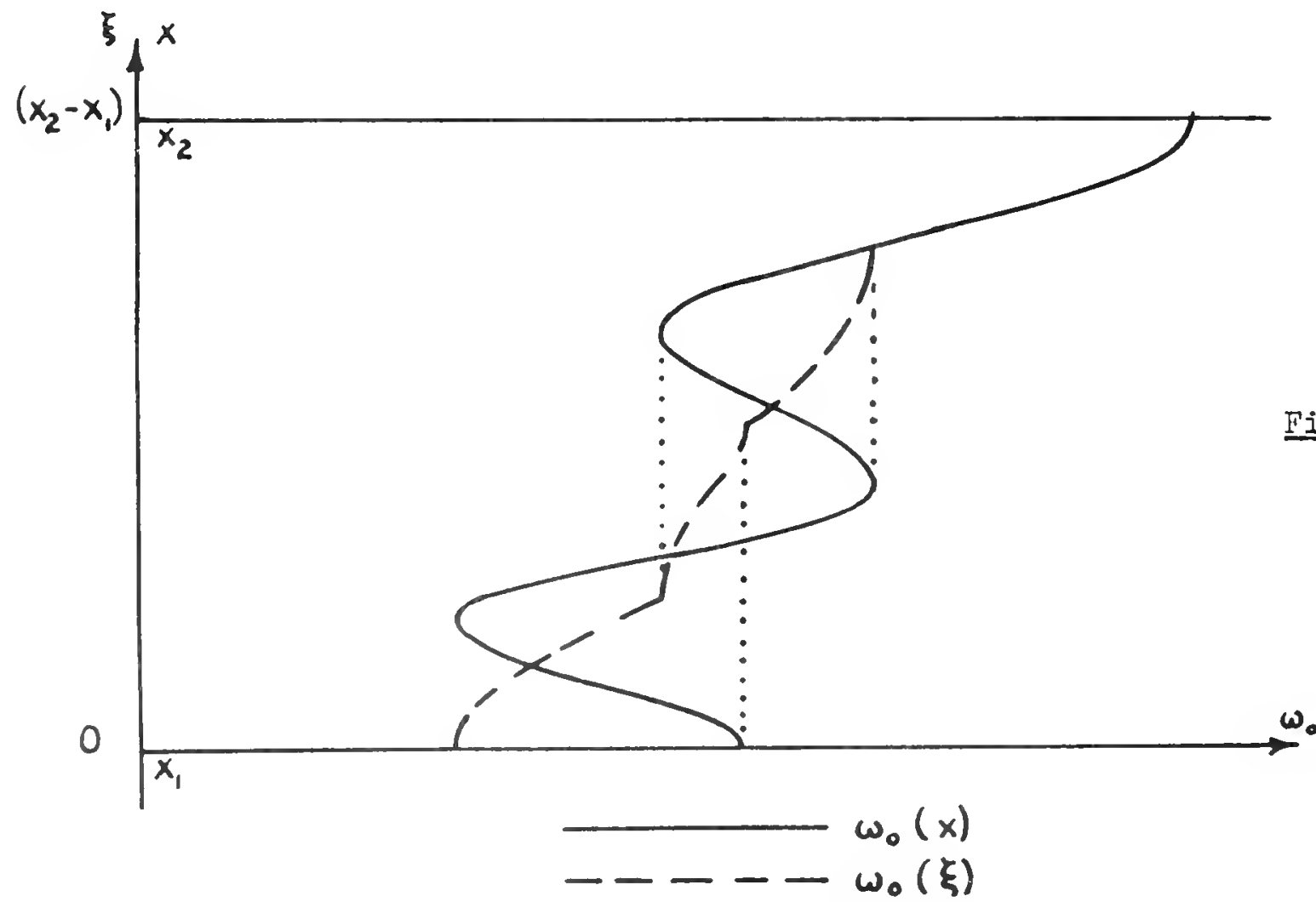


we assume that $\omega_{0}(x)$ is continuous and has continuous derivatives (as it would in an actual ionosphere), $\xi^{\prime}\left(\omega_{0}\right)$ is singular at all values of $\omega_{0}$ corresponding to maxima and minima of $\omega_{0}(x)$.

Since there is a family of distributions $\omega_{0}(x)$ characterized by the same width $\xi\left(\omega_{0}\right)$ and therefore yielding the same transit time $\tau(\omega)$, we cannot hope to calculate $\omega_{0}(x)$. All we can calculate is $\xi\left(\omega_{0}\right)$. This is more useful, however, than it might seem at first glance. From the behavior of $\xi\left(\omega_{0}\right)$ near the singularities of its derivative we can calculate all the minimum and maximum values of $\omega_{0}$ (but not their location), as well as the second derivatives of $\omega_{0}(x)$ at the extrema.

We now proceed to calculate $\omega_{0}(\xi)$ or $\xi\left(\omega_{0}\right)$. In terms of $\omega_{0}(\xi)$ the transit time is

$$
\tau_{1.2}=\frac{1}{c} \int_{0}^{x_{2}-x_{1}}\left[1-\omega_{0}^{2}(\xi) / \omega^{2}\right]^{-1 / 2} d \xi
$$

where $\omega_{0}(\xi)$ is a monotonically increasing function. At this point it is converient to introduce a number of transformations. Let

$$
\begin{aligned}
& E=\omega^{2}-\omega_{2}^{2}, \\
& V(\xi)=\omega_{2}^{2}-\omega_{0}^{2}(\xi), \\
& V_{0}=v(0)=\omega_{2}^{2}-\omega_{0}^{2}(0)=\omega_{2}^{2}-\omega_{3}^{2} .
\end{aligned}
$$

Furthermore, instead of dealing with the transit time $\tau_{12}$, let us subtract it from the time of travel over a distance $\left(x_{2}-x_{1}\right)$ of a pulse of the same frequency, w, in a homogeneous medium of plasma frequency $w_{2}$. Defining $T$ in this manner, we have 
(山)

$$
T(E)=\frac{1}{c} \int_{0}^{x_{2}-x_{1}} \cdot\left[-\sqrt{\frac{E+\omega_{2}^{2}}{E+V(\xi)}}+\sqrt{\frac{E+\omega_{2}^{2}}{E}}\right] d \xi
$$

or

$$
c\left(E+\omega_{2}^{2}\right)^{-1 / 2} T(E)=\int_{0}^{x_{2}-x_{1}}\left[E^{-1 / 2}-(E+V)^{-1 / 2}\right] d \xi
$$

Integrating by parts onco, obtain

$$
c\left(E+\omega_{2}^{2}\right)^{-I / 2} T(E)=\xi\left[E^{-I / 2}-(E+V)^{-I / 2}\right]_{\xi=0}^{\xi=x_{2}-x_{1}}+\frac{1}{2} \int_{0}^{x_{2}-x_{1}}(E+\nabla)^{-3 / 2} \frac{d \nabla}{d \xi} \xi d \xi .
$$

The first term drops out since $\xi$ vanishes at one end of the interval, while $\nabla$ vanishes at the other. Since $V(\xi)$ is monotonically decreasing we can make $V$ the variable of integration, so that

or

$$
c\left(E+\omega_{2}^{2}\right)^{-1 / 2} T(E)=\frac{1}{2} \int_{0}^{V_{0}} \xi(V)(E+V)^{-3 / 2} d V
$$

$$
2 c\left(E+\omega_{2}^{2}\right)^{-1 / 2} E^{3 / 2} T(E)=\int_{0}^{V_{0}} \xi(V)(I+V / E)^{-3 / 2} d V
$$

The function $\xi(V)$ has already been delined in the interval $0 \leq V \leq \nabla_{0}$. Furthermore $\xi\left(v_{0}\right)=0$. Hence we can arbitrarily define the function $\xi(v)$ for $V>v_{0}$ as being identically zero. 'ilth a definition of $\xi(V)$ so extended, we have the integral

$$
\text { equation } \quad f(I)=\int_{0}^{\infty} \xi(V)(I+V / E)^{-3 / 2} d V \text {, }
$$




$$
f(E)=2 c\left(E+\omega_{2}^{2}\right)^{-1 / 2} E^{3 / 2} T(E)
$$

In Eq. (18), $f(E)$ is given and we are to find $\xi(V)$.

The function $f(E)$ must, however, belong to the class of functions that can be derived from physically admissible functions $V(\xi)$. This imposes certain limitations on the function $f(E)$ from which the unknown $\xi(V)$ is to be calculated. For example, it is obvious from Eq. (18) that for increasingly large E, $f(E)$ must tend to a constant.

3. Solution of the integral equation

Application of the Mellin transform is suggested by the presence of a quotient kernel, and by the fact that both $E$ and $V$ range from 0 to $\infty$. The Mellin transform of a function $g(x)$ is defined as follows:

$$
\vec{g}(s)=\int_{0}^{\infty} g(x) x^{s-1} d x
$$

Let us consider a function

$$
f(y)=\int_{0}^{\infty} g(x) h\left(\frac{y}{x}\right) d x
$$

The Mellin transform of $f(x)$ is

$$
\bar{f}(s)=\int_{0}^{\infty} d y y^{s-1} \int_{0}^{\infty} d x g(x) h\left(\frac{Z}{x}\right)=\int_{0}^{\infty} d x g(x) x^{s} \int_{0}^{\infty} d \eta h(\eta) \eta^{s-1}=\bar{g}(s+1) \bar{h}(s) .
$$

In our problem the kernel $h$ is

$$
h(\eta)=\left(1+\frac{1}{\eta}\right)^{-3 / 2} \text {, }
$$




$$
\bar{L}(s)=\int_{0}^{\infty} \eta^{s+1 / 2}(1+\eta)^{-3 / 2} d \eta=\Gamma\left(s+\frac{3}{2}\right) \Gamma(-s) / \Gamma(3 / 2) .
$$

Thus we find the Mellin transform of the unknown function $\xi$ :

$$
\bar{\xi}(s)=\frac{\bar{f}(s-1)}{\bar{h}(s-1)}=\frac{\Gamma(3 / 2) \bar{f}(s-1)}{\Gamma(s+1 / 2) \Gamma(1-s)} .
$$

The function $\xi(V)$ can be obtained from $\bar{\xi}(s)$ by the use of the inverse Mellin transform.

4. Special values

We can deduce a muber of characteristics of the function $\omega_{0}(\xi)$ from the Mellin transform directly, without performing the inversion. Thus, for exarple, we can find a measure of total charge:

$$
\int_{0}^{x_{2}-x_{1}} \omega_{0}^{2}(\xi) d \xi=\left(x_{2}-x_{1}\right) \omega_{2}^{2}-\int_{0}^{x_{2}-x_{1}} v d \xi
$$

$$
=\left(x_{2}-x_{1}\right) \omega_{2}^{2}-\int_{0}^{\infty} \xi(\nabla) d V=\left(x_{2}-x_{1}\right) \omega_{2}^{2}-\xi(1) .
$$

If we try to evaluate $\vec{\xi}(I)$ from Eq. (24) we find that both the denominator and the mumerator of the fraction tend to infinity as $s \rightarrow 1$. Thus wave

$$
\int_{0}^{x_{2}-x_{1}} \omega_{0}^{2}(\xi) d \xi=\omega_{2}^{2}\left(x_{2}-x_{1}\right)-\lim _{\varepsilon \rightarrow 0} \frac{\bar{\rho}(\varepsilon)}{\Gamma(-\varepsilon)} .
$$

Carrying the process one step further we note that $\Gamma(x)$ has a simple pole at $x=0$, with residue 1 . Hence we can replace $\Gamma(-\varepsilon)$ by $-1 / \varepsilon$ in Eq. (26)s 
(27)

$$
\int_{0}^{x_{2}-x_{1}} \omega_{0}^{2}(\xi) d \xi=\left(x_{2}-x_{1}\right) \omega_{2}^{2}+\lim _{\varepsilon \rightarrow 0} \varepsilon \bar{f}(\varepsilon) .
$$

However, we can calculate the total charge in a much simpler way directly from $f(E)$. If we expand the kernel of the integral equation (18) in powers of (V/E), and retain the first term only, we obtain:

$$
f(E) \rightarrow \int_{0}^{\infty} \xi(V) d V \quad \text { as } \quad E \rightarrow \infty
$$

The above relation holds in the limit of large $\mathrm{E}$, since the kernel tends to unity. Thus have

$$
\int_{0}^{x_{2}-x_{1}} \omega_{0}^{2}(\xi) d \xi=\left(x_{2}-x_{1}\right) \omega_{2}^{2}-\lim _{E \rightarrow \infty} f(E) .
$$

The identity of - Iim $\varepsilon \vec{f}(\varepsilon)$ and lim $f(E)$ is not fortuitous, but can be

$$
\varepsilon \rightarrow 0 \quad E \rightarrow \infty
$$

demonstrated as follows. Consider the expression for $\varepsilon \overrightarrow{\mathbf{I}}$ :

$$
\varepsilon \bar{f}(\varepsilon)=\varepsilon \int_{0}^{\infty} E^{\varepsilon-1} f(E) d F=\varepsilon \int_{0}^{a} E^{\varepsilon-1} f(E) d E+\varepsilon \int_{a}^{\infty} E^{\varepsilon-1} f(E) d E .
$$

It can be shown that

$$
0<\mathrm{E}<\mathrm{a}, \quad|\mathrm{f}(\mathrm{E})|<\mathrm{bE},
$$

where $b$ is a positive constant and

$$
\text { (32) } E>\text { a, }
$$

$$
f(E)=\sum_{n=0}^{\infty} f_{n}\left(\frac{I}{E}\right)^{n}
$$

the series being uniformly and absolutely convergent. The first of the two 
integrals on the right-hand side of (30) is less than

$$
b \varepsilon \int_{0}^{a} E^{\varepsilon} d E=\frac{b \varepsilon}{\varepsilon+I} a^{\varepsilon+1}
$$

which tends to zero as $\varepsilon \rightarrow 0$. In the other integral we change the variable

$$
E=1 / x,
$$

so that

$$
\varepsilon \int_{a}^{\infty} E^{\varepsilon-1} f(E) d E=\varepsilon \int_{0}^{\frac{1}{2}} x^{-\varepsilon-1}\left(\sum_{n=0}^{\infty} f_{n} x^{n}\right) d x
$$

$$
=\varepsilon \sum_{n=0}^{\infty} f_{n} \frac{a^{\varepsilon-n}}{n-\varepsilon} \rightarrow-f_{0}, \quad \text { as } \varepsilon \rightarrow 0 \text {, }
$$

and we see that two limits are indeed identical. The Identity is useful for checking purposes.

Suppose that for small $\xi, V(\xi)$ can be described approximately by

$$
V(\xi)=V_{0}\left(1-A \xi^{a}\right)
$$

From the definition of the Mellin transform it follows that for large 8 the transform of $\xi$ will depend only on the behavior of $\xi(V)$ near the maximum of $V$, and has the form

$$
\bar{\xi}(8) \approx v_{0}^{s} A^{-1 / \alpha} s^{-(1 / \alpha+1)}, \quad s \rightarrow \infty
$$

Thus from the behavior of $\bar{\xi}(s)$ for large values of 8 we can determine $\nabla_{0}, A$, and a. In the case of an actual ionospheric distribution, $\alpha=2$, so that only $V_{0}$ and A would have to be determined. 
$-13-$

5. Conclusion

We see that we can calculate the electron density distribution from the transit time. The answer is not unique but the ambiguity is inherent in the problem and not due to the method used.

Th not serious defect of the method for pretical calculations sat integrals mot be evaluated over an infinite interval, and that the integrand, and hence the travel tim $\tau_{12}$, must be specteled over the whole infinite range. The problem of developing suitable approximate methods, utilizing the travel time at a finite number of frequencies, has not been tackled here. It would be necessary to solve this problem before applying the method developed bore to empirical data. 


\section{Appendix}

Motion of a particle in a potential well

In the theory of the motion of a particle through a potential well, in one dimension, the following problem occurs which is essentially the same as the one discussed in this paper.

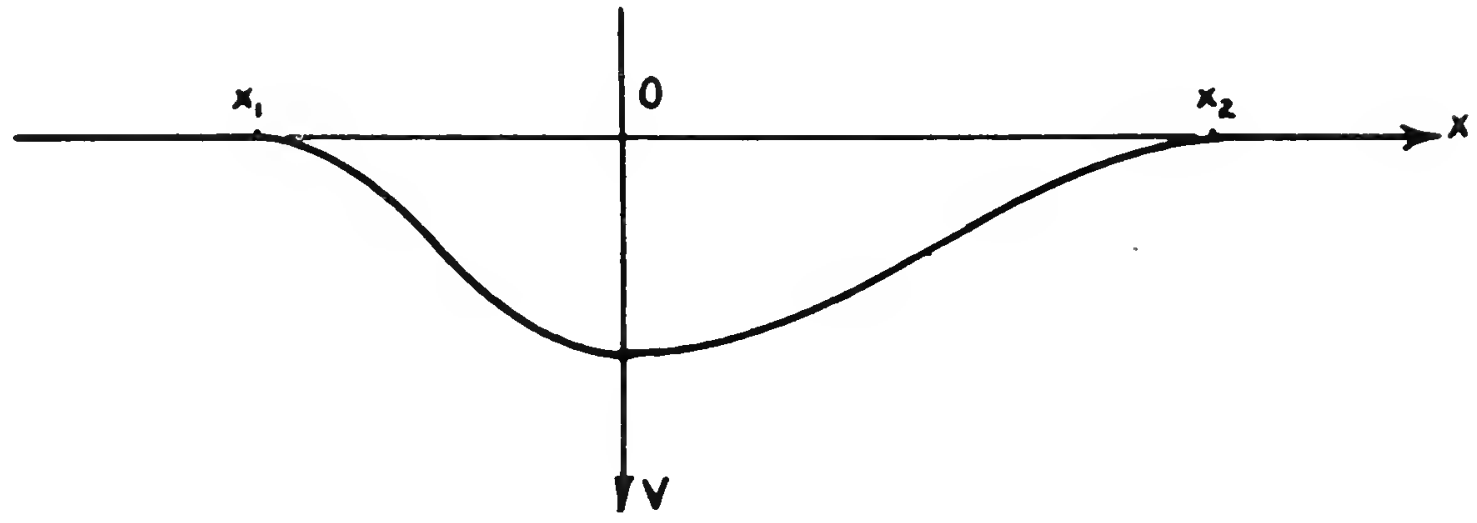

\section{Figure 5}

Let us consider a particle of mass $m$, having energy $E$, moving in a potential well of depth $v(x)$. The velocity of the particle is then

$$
v=(2 / m)^{1 / 2} \cdot(E+V)^{1 / 2}
$$

and the time of travel from $x_{1}$ to $x_{2}$ is

$$
\tau_{12}(E)=\int_{x_{1}}^{x_{2}} \frac{d x}{\nabla(x, E)}=(m / 2)^{1 / 2} \int_{x_{1}}^{x_{2}}(E+V)^{-l / 2} d x .
$$

We see that here again we encounter the same lack of untqueness as in the ionospheric case. We can again define $\xi$ as the width of the well at the depth V; for all wells having the same $V(\xi)$ the transit time is 
(A.3)

$$
\tau_{12}(E)=(m / 2)^{1 / 2} \int_{0}^{x_{2}-x_{1}}[E+\nabla(\xi)]^{-1 / 2} d \xi .
$$

Again, as in the preceding case, it is more convenient to deal with the difference between the transit time in the absence of a potential and the transit time $\tau_{12}$, rather than with $\tau_{12}$ directly. Let us define that difference, $T:$

(A.4) $\quad T(E)=(m / 2)^{1 / 2} \int_{0}^{x_{2}-x_{1}}\left[E^{-1 / 2}-(E+V)^{-1 / 2}\right] d \xi$.

Finally, again by integration by parts, we obtain

$$
(2 / m)^{I / 2} T(E)=\frac{1}{2} \int_{0}^{V_{0}} \xi(V)(E+V)^{-3 / 2} d V
$$

which gives an integral equation identical with (18) if we set

$$
f(E)=2(2 / m)^{1 / 2} E^{3 / 2} T(E) \text {. }
$$

In the case of potential well, the potential $\nabla(\xi)$ may not vanish identically for $\xi>\left(x_{2}-x_{1}\right)$, but tend to zero as $\xi$ increases. This has no effect on the formulation of the problem so long as $V$ tends to zero faster than $\xi^{-1}$. In mand probleas we know a priori that the potential well is symmetric. In these cases, if $V(x)$ 1s monotonically increasing from $-\infty$ to 0 , then it $c a n$ be calculated uniquely.

\section{References}

[1] Manning, L.A., Determination of ionospheric electron distribution; Froc. IRE 35, 1203-7 (1947).

[2] Manning, L.A., The reliability of ionospheric belght determinations; Proc. IRE 37, 599-603 (1949). 


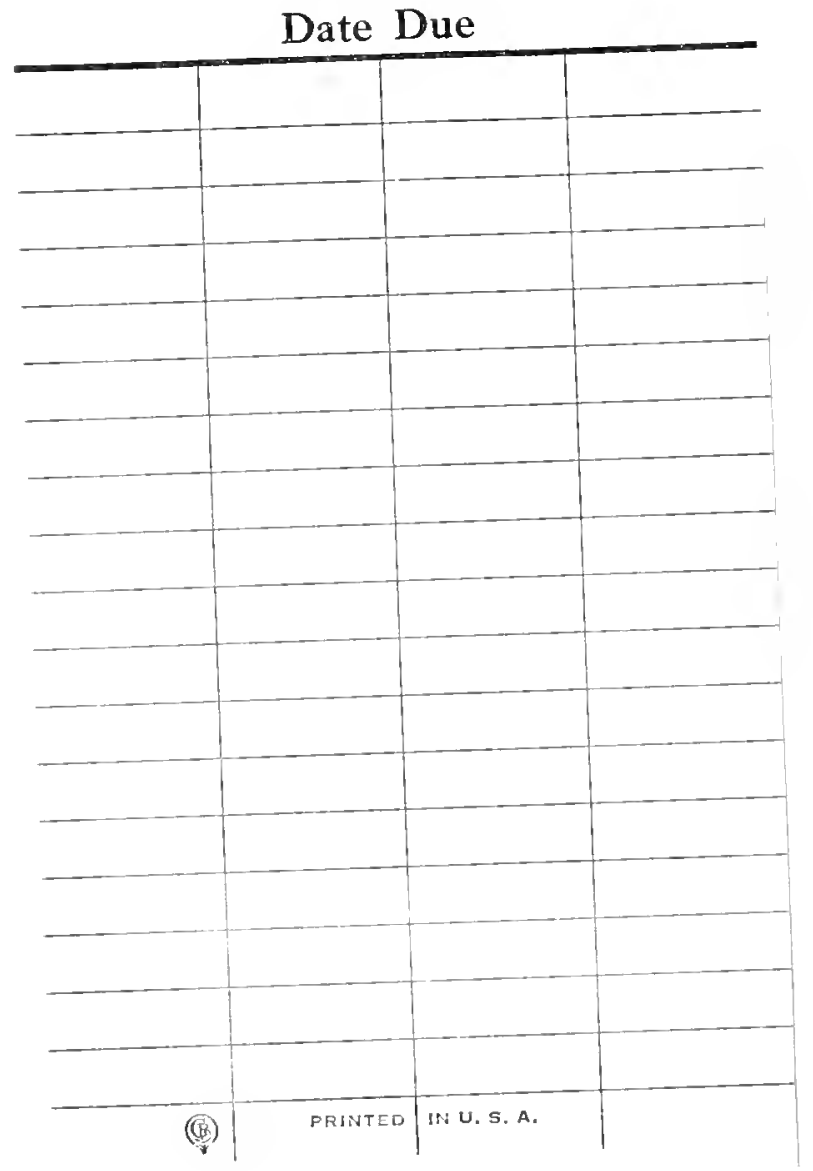




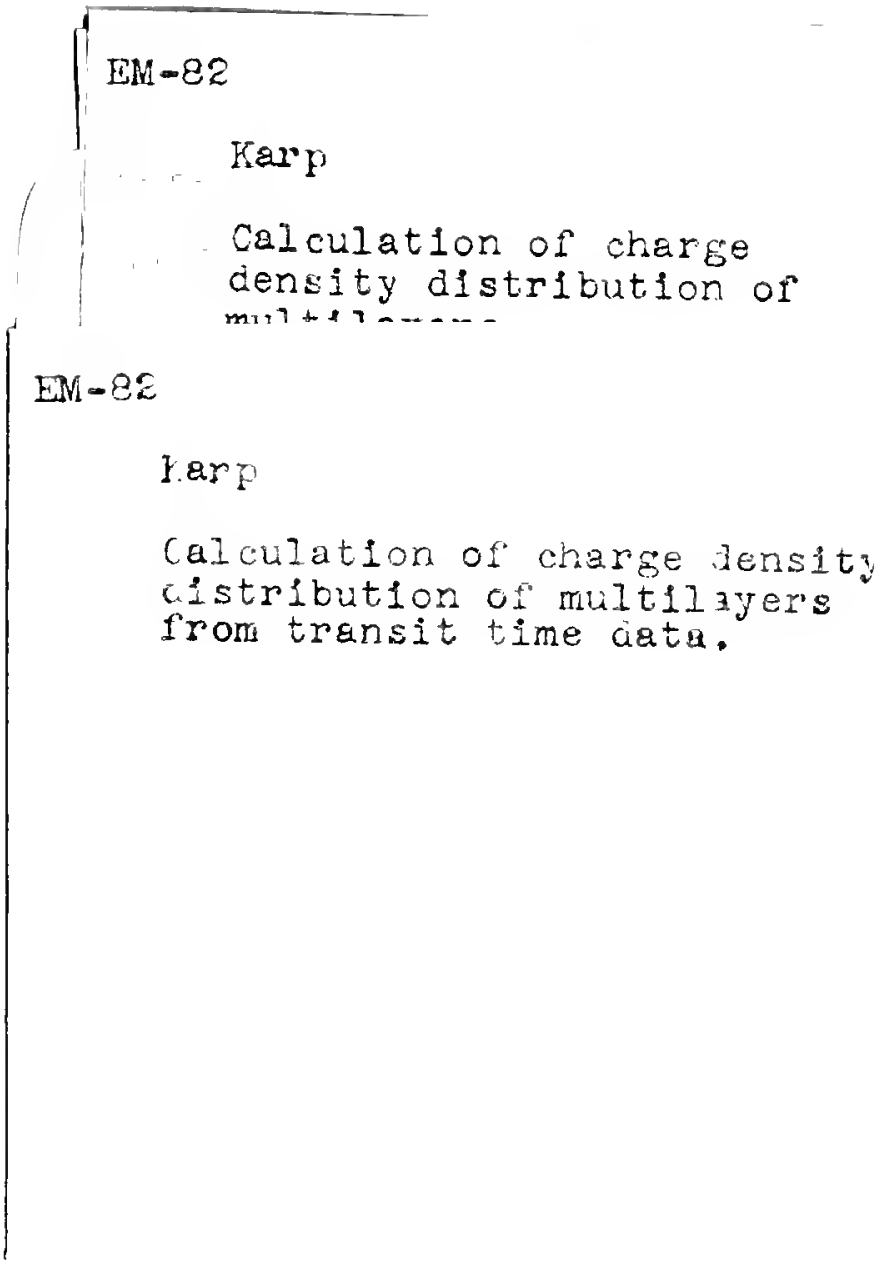


